\title{
Nodulação em plantas de soja (Glycine max L. Merril) submetidas a diferentes adubações
}

\section{Nodulation in soybean plants (Glycine max L. Merril) submitted to different fertilizations}

\author{
Diego Ferreira da Silva ${ }^{(1)}$; Erikson Kadoshe de Morais Raimundo ${ }^{(\mathbb{D}}{ }^{2}$; Victor Augusto Forti ${ }^{(\mathbb{D}}{ }^{3}$
}

${ }^{1}$ Graduado em Agronomia, Universidade Federal de São Carlos, Araras, São Paulo, (16) 99620-2934, diegoagrono@ gmail.com; ${ }^{2}$ Mestrando em Agroecologia e Desenvolvimento Rural, Universidade Federal de São Carlos, Araras, kadoshetecagro@ hotmail.com; ${ }^{3}$ Doutorado em Fitotecnia pela Universidade de São Paulo, Professor de Ensino Superior da Universidade Federal de São Carlos, Araras, viaugu@ yahoo.com.br.

N O T A

C I E N T Í F I C A

Recebido: $27 / 04 / 2018$

Aprovado: 23/06/2019

\section{Palavras-chave:}

Inoculação

Simbiose

Produção orgânica

Key words:

Inoculation

Symbiosis

Organic production

\section{R E S U M O}

Devido a sua capacidade de fixação biológica de nitrogênio, a cultura da soja tem apresentando maiores produtividades com a inoculação de bactérias do gênero Rhizobium. Entretanto, a inoculação pode ser influenciada por diversos fatores, inclusive pelo tipo de adubação realizada. Este trabalho teve por objetivo avaliar a influência de diferentes adubações na nodulação e no desenvolvimento de plantas de soja. Para isso, testaram-se as adubações orgânicas, com base em esterco bovino e cama de frango, a adubação química e um tratamento testemunha, sendo eles com ou sem inoculação de Bradyrhizobium japonicum. Foram avaliadas as variáveis de altura de planta, número de folhas, massa da matéria seca de planta, número de nódulos e massa da matéria seca dos nódulos. Os tratamentos com adubação de esterco bovino apresentaram os melhores resultados em todas as variáveis analisadas, com ou sem a inoculação de sementes. O esterco bovino se apresenta como uma excelente escolha para a adubação de soja, uma vez que possibilita um maior desenvolvimento de parte aérea, sistema radicular e número de nódulos, evidenciando que poderá contribuir para um maior rendimento no final do ciclo da cultura.

A B S T R A C T
Due to its biological nitrogen fixation capacity, soybean cultivation has shown higher yields
with the inoculation of Rhizobium sp. bacteria. However, the inoculation process can be
influenced by several factors, including the fertilization performed. The aim of this research
was to evaluate the influence of different fertilizations on nodulation and development of
soybean plants. For this, the organic fertilizations, based on cattle manure and poultry litter,
chemical fertilization and a control treatment were tested, with or without Bradyrhizobium
japonicum inoculation. It was evaluated the plant height, leaf number, plant dry matter and
the number and dry matter of nodules. The treatments with cattle manure showed the best
results in all variables, with or without seed inoculation. Cattle manure is an excellent
choice for soybean fertilization, since it allows a greater plant development and a higher
number of nodules contributing to a higher yield at the end of the crop cycle.

\section{INTRODUÇÃO}

A cultura da soja (Glycine max L. Merril) é uma das mais importantes para a economia nacional e mundial. Segundo a Conab (2018) estima-se que a produção de soja no Brasil nos anos de 2018 e 2019 pode chegar a cerca de 120 milhões de toneladas. Devido a sua capacidade de fixação biológica de nitrogênio (FBN) a cultura da soja tem ganhado ainda mais destaque no Brasil, apresentando produções mais eficiente com a inoculação de cepas de Bradyrhizobium japonicum, suprindo a sua demanda nutricional e favorecendo um melhor desenvolvimento de plantas e produção (BÁRBARO et al., 2009; HUNGRIA et al., 2013).
Isso é possível graças associação da planta com bactérias do gênero Rhizobium favorecendo a ação simbiótica no sistema radicular, que por sua vez, reflete diretamente na FBN (PAMPANA et al., 2016). A FBN é realizada por microrganismos diazotróficos que possuem um complexo enzimático responsável pela redução do $\mathrm{N}_{2}$ atmosférico à amônia (ZILLI et al., 2013), tornando-o disponível para serem assimilados pelas plantas. $\mathrm{O}$ nitrogênio $(\mathrm{N})$ é essencial para o desenvolvimento e manutenção de plantas, inclusive para soja. Estima-se que para produzir $1.000 \mathrm{~kg}$ de grãos, são necessários $80 \mathrm{~kg}$ de N (HUNGRIA et al., 2007).

\section{Revista Verde}

ISSN 1981-8203

Pombal, Paraíba, Brasil v. 14 , n.3, jul.-set, p.470-475, 2019 doi: 10.18378/rvads.v14i3.6501 
Além disso, a inoculação de sementes com estirpes de $B$. japonicum permite a redução de investimentos em adubos nitrogenados no processo produtivo. De acordo com Hungria et al. (2007), o Brasil economizou 6,6 bilhões de dólares com a redução da adubação nitrogenada, quando realizada a inoculação das sementes, fato que levou o Brasil a um lugar de destaque no cenário mundial.

Para muitas culturas, como é o caso da soja, promover essa associação simbiótica é essencial para garantir altas produtividades em campos de produção. Entretanto, diversos fatores podem interferir na nodulação das plantas, tais com: fertilidade do solo (MELCHIORRE et al., 2010), disponibilidade de fósforo (SOARES et al., 2016), $\mathrm{pH}$ (SOUZA et al., 2010) e microbiota do solo (PEREIRA et al., 2013), os quais podem ser diretamente influenciados pelo tipo de adubação.

De maneira geral, a fertilização com estercos de origem animal pode melhorar as características físicas, químicas e biológicas do solo (CARDOSO et al., 2011), propiciando maior retenção de água, maior agregação das partículas do solo, redução do processo erosivo, aumento da taxa de infiltração de água e menor amplitude térmica do solo. A utilização de adubos orgânicos como a cama de frango e o esterco bovino permite uma maior diversidade de microrganismos (FALCÃO et al., 2013), que podem contribuir com a nodulação. Ragagnin et al. (2013), relataram que a utilização da cama de frango na adubação de plantas de soja propiciou um aumento significativo no número de nódulos. Carvalho et al. (2011) trabalhando com adubação em soja com cama de frango e fertilizante mineral, evidenciaram que a adubação orgânica apresentou melhores resultados na altura de plantas, massa de grãos, número de vargens por plantas e rendimento de grãos.

A adubação nitrogenada, quando realizada em leguminosas, podem tem efeitos adversos na FBN, devido a diminuição da respiração nodular e a limitação de carboidratos no metabolismo do nódulo (SILVA et al., 2011; XAVIER et al., 2008). De acordo com Hungria et al. (2001), não é necessário realizar esse método de adubação para a cultura, desde de que a mesma tenha sido inoculada com bactérias que favoreça a FBN. Braccini et al. (2016) investigando o efeito de diferentes tipos de inoculação na cultura da soja, evidenciaram que a inoculação por si só apresenta resultados mais satisfatórios do que quando aliada a adubação com fertilizantes nitrogenados.

Desta maneira, o objetivo deste estudo foi avaliar a influência de diferentes adubações na nodulação e desenvolvimento de plantas de soja.

\section{MATERIAL E MÉTODOS}

O trabalho foi conduzido na área experimental e no Laboratório de Agricultura Orgânica - LAO do Centro de Ciências Agrárias da Universidade Federal de São Carlos (CCA/UFSCar), nas coordenadas geográficas $28^{\circ} 18^{\prime} 26^{\prime \prime} \mathrm{S}$; $47^{\circ} 22$ '42” O.

O solo utilizado foi o Latossolo Vermelho Distrófico, o qual foi submetido a análise química (Tabela 1) para proceder com a correção da acidez de acordo com as recomendações técnicas da cultura descritas por Aguiar et al. (2014), aplicando-se $26,21 \mathrm{~g}$ de calcário por vaso.

Tabela 1. Análise química do solo Latossolo Vermelho Distrófico.

\begin{tabular}{cccccccccc}
\hline $\mathrm{pH}$ & $\mathrm{M} . \mathrm{O}$ & $\mathrm{P} \mathrm{Resina}$ & $\mathrm{H}+\mathrm{AL}$ & $\mathrm{SB}$ & $\mathrm{K}$ & $\mathrm{Ca}$ & $\mathrm{Mg}$ & $\mathrm{CTC}$ & $\mathrm{V}$ \\
\hline$\left(\mathrm{CaCl}_{2}\right)$ & $\left(\mathrm{g} / \mathrm{dm}^{3}\right)$ & $\left(\mathrm{mg} / \mathrm{dm}^{3}\right)$ & & & $\mathrm{mmolc} / \mathrm{dm}^{3}$ & & & \\
5 & 33 & 5 & 33 & 16,6 & 2,6 & 8 & 6 & 49,6 & 33 \\
\hline
\end{tabular}

Foram utilizados vasos com capacidade de 20 litros, nos quais, foram considerados como tratamentos, três diferentes adubações, compostas de cama de frango, esterco bovino e adubo químico, acrescido de uma testemunha, com quatro repetições compostas de duas plantas cada.

Foram acrescidos ao solo nos vasos cerca de $126 \mathrm{~g} / \mathrm{vaso}$ de cama de frango (aproximadamente 2,5 t/ha), $189 \mathrm{~g} / \mathrm{vaso}$ de esterco bovino (aproximadamente 3,8 t/ha) e 3,26 g/vaso (650 $\mathrm{kg} / \mathrm{ha}$ ) de fertilizante mineral da formulação 8-20-15, com base nas recomendações de adubação da cultura (VAN RAIJ et al., 1997). Os adubos foram homogeneizados ao solo para acelerar o processo de mineralização dos mesmos.

As fontes orgânicas foram previamente analisadas, determinando-se a cama de frango composta de 4,21, 3,38 e $2,65 \%$ de $\mathrm{P}_{2} \mathrm{O}_{5}, \mathrm{~K}_{2} \mathrm{O}$ e N, respectivamente e o esterco bovino, com 6,91, 2,20 e 1,70\% de $\mathrm{P}_{2} \mathrm{O}_{5}, \mathrm{~K}_{2} \mathrm{O}$ e N, respectivamente.

O cultivar utilizado foi a BRS 7380RR, previamente inoculada ou não com estirpe de $B$. japonicum, com $7 \times 10^{9}$ ufc/ml, na dose de $80 \mathrm{ml}$ para $50 \mathrm{~kg}$ de sementes. As sementes foram inoculadas com o auxílio de um saco plástico higienizado, homogeneizando manualmente por dois minutos com movimentos rotacionais. Após a inoculação, foi realizado o plantio, distribuindo 4 sementes em cada vaso. A capina foi realizada manualmente no decorrer do experimento e a irrigação foi realizada uma vez por semana considerando $1.350 \mathrm{ml}$ por vaso, $60 \%$ da capacidade de campo do solo utilizado. Os vasos foram mantidos sob cobertura de telhado até o $40^{\circ}$ dia após a semeadura.

Após esse período, foram avaliadas as variáveis de número de nódulos, e comprimento de raiz, mensurado do colo da planta até o final da raiz central. Para a altura de plantas, considerou-se a distância entre o solo e a extremidade apical da haste principal, e para o número de folhas, contabilizaram-se todas as folhas trifolioladas expandidas. Para a obtenção da massa de matéria seca de nódulos, raiz e parte aérea, o material foi acondicionado separadamente em estufa de circulação forçada a $65^{\circ} \mathrm{C}$ até a determinação da massa constante.

$\mathrm{O}$ experimento foi instalado em delineamento inteiramente casualizado, em esquema fatorial $4 \times 2$, quatro tratamentos de adubação (cama de frango, esterco bovino, adubação química e testemunha) e dois de inoculação (com e sem inoculante). Para cada combinação foram instaladas quatro repetições, compostas de duas plantas cada. Os dados foram inicialmente analisados por meio da análise de variância e, quando efeitos significativos, foram analisados pelo teste de Tukey a $5 \%$ de probabilidade com o auxílio do programa estatístico SISVAR (FERREIRA, 2011).

\section{RESULTADOS E DISCUSSÃO}

Para as variáveis de número de nódulos e massa de matéria seca de nódulos (Tabela 2) os tratamentos com cama de frango e testemunha apresentam maior média para plantas 
inoculadas em relação aos mesmos tratamentos sem inoculação e, consequentemente uma maior massa de nódulos quando as sementes foram inoculadas. Para os tratamentos de esterco bovino e adubação química, não foram observados efeitos da inoculação no número de nódulos. Ainda assim, quando comparadas apenas as adubações, o tratamento utilizando esterco bovino apresenta média superior a adubação química com ou sem a inoculação de sementes. A adubação também interferiu na massa de matéria seca dos nódulos, sendo que a adubação química quando utilizada em sementes inoculadas apresenta melhores resultados, contrário ao observado para esterco bovino.

Tabela 2: número de nódulos (NN) e massa de matéria seca de nódulo (MMSN), em gramas, de plantas de soja submetidas a adubações com esterco bovino, cama de frango, adubo químico e testemunha, com (C) e sem (S) inoculação com $B$. japonicum.

\begin{tabular}{|c|c|c|c|c|}
\hline \multirow{2}{*}{ Trat. } & \multicolumn{2}{|c|}{$\mathrm{NN}$} & \multicolumn{2}{|c|}{ MMSN (g) } \\
\hline & $\mathrm{C}$ & $S$ & $\mathrm{C}$ & $S$ \\
\hline E. Bovino & $10,8 \mathrm{aA}$ & $8,9 \mathrm{aA}$ & $0,0113 \mathrm{aB}$ & $0,0179 \mathrm{aA}$ \\
\hline C. Frango & $6,9 \mathrm{bA}$ & $3,8 \mathrm{bB}$ & $0,0061 \mathrm{bA}$ & $0,0029 \mathrm{bB}$ \\
\hline A. Químico & $6,1 \mathrm{bA}$ & $5,0 \mathrm{bA}$ & $0,0052 \mathrm{bcA}$ & $0,0027 \mathrm{bB}$ \\
\hline Testemunha & $8,1 \mathrm{abA}$ & $4,3 \mathrm{bB}$ & $0,0042 \mathrm{cA}$ & $0,0023 \mathrm{bB}$ \\
\hline $\mathrm{CV}$ & \multicolumn{2}{|c|}{23,04} & \multicolumn{2}{|c|}{14,31} \\
\hline
\end{tabular}

Médias seguidas da mesma letra minúscula nas colunas e maiúscula nas linhas, para cada variável, não diferem entre si pelo teste de Tukey a 5\% de probabilidade.

De acordo com Fagan et al. (2007), plantas de soja submetidas a adubação nitrogenada tem redução significativa no desenvolvimento de nódulos, contrariando os dados deste trabalho, em que foi observado ausência de efeito da inoculação no número de nódulos quando as plantas foram mantidas sob adubação química.

O tratamento submetido a adubação com esterco bovino é superior aos demais, apresentando superioridade de até $778 \%$ a mais de acumulo de MMSN se comparado com a testemunha. Já para o tratamento testemunha, observa-se uma diferença significativa entre a presença e ausência de inoculante na MMSN, na ordem de duas vezes mais na presença tratamento de sementes, o que evidencia o rendimento ocasionado pela inserção do B. japonicum e seu potencial para cultura da soja. Assim, observa-se que a o método de adubação com esterco bovino propicia o aumento na nodulação favorecendo o aporte de $\mathrm{N}$ para a planta, sendo dispensável até mesmo a inoculação de sementes. Fato visível, uma vez que se comparado ao tratamento testemunha, o tratamento com esterco bovino, quando inoculado, apresentou um aumento de $0,0071 \mathrm{~g}$ na matéria seco de nódulos. Já para os tratamentos com sementes inoculadas o tratamento com esterco bovino apresentou um acréscimo de 0,0156 g se comparado ao tratamento testemunha.

A adubação com esterco bovino provavelmente influenciou na melhoria das características físicas, químicas e biológicas do solo o que pode ter favorecido o processo de nodulação, seja por conta da maior biodiversidade de microrganismos no solo ou pela melhor condição edáfica propiciada para a ocorrência da simbiose.

$\mathrm{Na}$ fase inicial, entre 10 e 15 dias pós emergência, devem ser encontrados cerca de 4 a 8 nódulos por planta e nos estádios de florescimento (R1 e R2), em plantas bem noduladas, devem apresentar de 15 a 30 nódulos por sistema radicular (VARGAS; HUNGRIA, 1997). Neste trabalho as plantas avaliadas já se encontravam no estádio de florescimento pleno, no entanto apresentando número de nódulos reduzidos. Isso possivelmente está atrelado ao fato de as plantas terem sido cultivadas em época de semeadura inadequada para a cultivar afetando diretamente no fotoperíodo. Esse fato altera significativamente os estádios de desenvolvimento e produtividade (ALCÂNTARA NETO et al., 2012)

Para as variáveis comprimento de raiz e massa de matéria seca de raiz, não houve diferença entre os tratamentos com e sem inoculação (Tabela 3). No entanto, no que se refere a adubação, o tratamento submetido ao esterco bovino apresentou maior desenvolvimento no comprimento das raízes quando comparado com as outras adubações com inoculação e quando comparado ao adubo químico sem inoculante. Neste caso, a associação entre as adubações e a inoculação de semente com estirpes de B. japonicum, não interferiu no desenvolvimento do sistema radicular da planta, diferindo dos resultados apresentados por Braccini et. al. (2016), os quais afirmam que a inoculação com B. japonicum via tratamento de sementes, pode potencializar o maior crescimento do sistema radicular.

Tabela 3: Comprimento de raiz (CR), em cm, e massa de matéria seca do sistema radicular (MMSR), em gramas, de plantas de soja submetidas a diferentes adubações com esterco bovino, cama de frango, adubo químico e testemunha com (C) e sem (S) inoculação com B. japonicum.

\begin{tabular}{|c|c|c|c|c|}
\hline \multirow{2}{*}{ Trat. } & \multicolumn{2}{|c|}{$\mathrm{CR}(\mathrm{cm})$} & \multicolumn{2}{|c|}{ MMSR (g) } \\
\hline & $\mathrm{C}$ & $S$ & $\mathrm{C}$ & $S$ \\
\hline E. Bovino & $54,62 \mathrm{aA}$ & $49,50 \mathrm{aA}$ & $0,62 \mathrm{aA}$ & $0,59 \mathrm{aA}$ \\
\hline C. Frango & $39,00 \mathrm{bA}$ & $41,50 \mathrm{abA}$ & $0,39 \mathrm{bA}$ & $0,46 \mathrm{aA}$ \\
\hline A. Químico & $41,25 \mathrm{bA}$ & $37,56 \mathrm{bA}$ & $0,37 \mathrm{bA}$ & $0,29 \mathrm{bA}$ \\
\hline Testemunha & $40,50 \mathrm{bA}$ & $45,88 \mathrm{abA}$ & $0,36 \mathrm{bA}$ & $0,31 \mathrm{bA}$ \\
\hline $\mathrm{CV}$ & \multicolumn{2}{|c|}{11,99} & \multicolumn{2}{|c|}{16,84} \\
\hline
\end{tabular}

Médias seguidas da mesma letra minúscula nas colunas e maiúscula nas linhas, para cada variável, não diferem entre si pelo teste de Tukey a 5\% de probabilidade. 
Um fator que pode estar inteiramente ligado a estes resultados é a quantidade de fósforo presente nos adubos orgânicos. O fósforo é um nutriente muito importante para o desenvolvimento da planta, atua em diversos processos metabólicos, constituição de ATP, de enzimas fosforilase e no desenvolvimento de plântulas e raízes, principalmente na fase inicial (SANTOS et al., 2015). O esterco bovino tem uma grande quantidade de $\mathrm{P}$ em sua composição $(6,91 \%)$, deste modo, a alta quantidade deste nutriente pode ter sido um dos fatores responsáveis pela diferença entre os tratamentos. Além disso, esse resultado pode estar também aliado com a boa estruturação do solo, uma vez que o esterco bovino atua melhorando as características do mesmo.

Outro fator importante é a presença de cálcio no esterco bovino. Segundo Raij et al. (1997), o esterco bovino apresenta cerca de $20 \mathrm{~g} / \mathrm{kg}$ de cálcio e, este macronutriente atua no crescimento radicular e em proteínas mensageiras de sinais químicos. Assim, é possível que a presença de cálcio no esterco bovino, juntamente com a calagem, auxiliou no desenvolvimento radicular, obtendo maiores médias.

Para a massa seca de raízes, também não foi observado efeito do tratamento de inoculação independentemente da adubação realizada. Por outro lado, ao se comparar isoladamente as adubações, observa-se que o tratamento com esterco bovino apresenta médias superiores aos demais tratamentos, com exceção da cama de frango na ausência da inoculação. Isso demonstra a real importância da adubação orgânica mesmo na ausência do tratamento de sementes na semeadura.

A possível explicação para este fato é que a matéria orgânica do solo pode ter contribuído de forma direta, fornecendo os nutrientes essenciais para o desenvolvimento das raízes e indireta, melhorando a qualidade do solo, possibilitando a ação de microrganismos e ocasionando maior desenvolvimento radicular.

Em relação à altura de plantas (Tabela 4), não foi observado diferença entre plantas inoculadas e não inoculadas, independentemente do tratamento de adubação, exceto para o adubo químico em que o tratamento sem inoculação promoveu aumento em relação ao mesmo tratamento com inoculação. Pedrozo, Oliveira e Alberton (2018) apresentaram resultados diferentes quanto a aplicação do mesmo inoculante, observando que plantas de soja inoculadas com B. japonicum tiveram aumento de até $67 \%$ se comparadas a testemunha

Tabela 4: Altura de plantas (AP), em cm, número de folhas (NF) e massa de matéria seca de parte aérea (MMSPA), em gramas, de plantas de soja submetidas a diferentes adubações com esterco bovino, cama de frango, adubo químico e testemunha com (C) e sem (S) inoculação com B. japonicum.

\begin{tabular}{ccccccc}
\hline \multirow{2}{*}{ Trat. } & \multicolumn{3}{c}{ AP } & \multicolumn{2}{c}{ NF } & \multicolumn{2}{c}{ MMSPA } \\
\cline { 2 - 7 } & C & S & C & S & C \\
\hline E. Bovino & $45,38 \mathrm{aA}$ & $45,50 \mathrm{aA}$ & $5,3 \mathrm{aA}$ & $5,6 \mathrm{aA}$ & $3,14 \mathrm{aA}$ & $3,33 \mathrm{aA}$ \\
C. Frango & $42,88 \mathrm{aA}$ & $40,38 \mathrm{abA}$ & $4,8 \mathrm{abA}$ & $5,0 \mathrm{aA}$ & $2,51 \mathrm{bA}$ & $2,16 \mathrm{bB}$ \\
A. Químico & $31,37 \mathrm{bB}$ & $39,75 \mathrm{abA}$ & $4,1 \mathrm{bcA}$ & $3,4 \mathrm{bB}$ & $1,41 \mathrm{cA}$ & $1,31 \mathrm{cA}$ \\
Testemunha & $31,25 \mathrm{bA}$ & $35,63 \mathrm{bA}$ & $3,8 \mathrm{cA}$ & $3,3 \mathrm{bB}$ & $1,31 \mathrm{cA}$ & $1,20 \mathrm{cA}$ \\
\hline CV & \multicolumn{3}{c}{10,03} & \multicolumn{3}{c}{9,27} \\
\hline
\end{tabular}

Médias seguidas da mesma letra minúscula nas colunas e maiúscula nas linhas, para cada variável, não diferem entre si pelo teste de Tukey a $5 \%$ de probabilidade.

Bossolani et al. (2018) relatam que plantas de soja quando inoculada com $B$. japonicum tiveram influência direta no aumento da altura das plantas. As sementes que foram tratadas com inoculante, após receberem a dose de fertilizante mineral, podem ter sofrido alteração na população de rizóbios, diminuindo o potencial de fixação pela não promoção da inoculação refletindo consequentemente na altura das plantas. A adição de adubos nitrogenados tem efeitos adversos na FBN em leguminosas. Silva et al. (2011) evidenciaram que doses altas de fertilizantes nitrogenados podem interferir na nodulação de plantas de soja.

Em relação à adubação, observa-se que nas plantas sem qualquer tipo de adubação (testemunha) a altura de planta foi inferior que nos demais tratamentos, não diferindo do adubo químico com inoculação e do adubo químico e cama de frango sem inoculação (Tabela 4). Dessa maneira, observa-se que, em ambos os tratamentos de inoculação, o tratamento com esterco bovino promoveu um aumento na altura de plantas de soja. É provável que se mantidas até o final do ciclo, as plantas poderiam apresentar resultados diferentes no que se refere à altura de plantas, uma vez que isso possibilitaria um maior aparecimento de nódulos e consequentemente um maior desenvolvimento de plantas.

No que se refere a número de folhas, os tratamentos submetidos a adubação química e o testemunha foram significativamente inferiores aos demais quando comparados entre as adubações. No entanto, quando comparados com ou sem inoculação, o tratamento inoculado favoreceu o aumento do número de folhas.

O nitrogênio é um nutriente essencial para o crescimento vegetativo, uma vez que é um componente fundamental na formação de bases nitrogenadas, fitohormônios e proteínas que compõe o tecido vegetal (NUNES et al., 2016; CANATTO et al., 2013). Desta forma a baixa disponibilidade de nitrogênio pode ter afetado diretamente o desenvolvimento das folhas. Os tratamentos submetidos a adubação com esterco bovino e cama de frango, com e sem inoculante possibilitaram uma melhor FBN e consequentemente uma melhor translocação $\mathrm{N}_{2}$ para as folhas aumentando sua arquitetura. Além disso, esses adubos orgânicos possuem um grande aporte de P e K. De acordo com Pereira et al. (2016), doses de potássio na cultura da soja, mostram respostas significativas para as variáveis altura de planta e NF uma vez que uma das principais funções do nutriente na planta é atuar como ativador enzimático e regulador da pressão osmótica auxiliando diretamente na formação das folhas. De acordo com Amaro (2007), em média, o esterco bovino e a cama de frango possuem 1,4 e $2,0 \%$ de $\mathrm{K}_{2} \mathrm{O}$ respectivamente. $\mathrm{O}$ esterco bovino e a cama de frango utilizadas para a adubação da soja neste trabalho possuíam 2,65 e 2,20\% 
respectivamente. Fato que pode ter contribuído para um melhor resultado no número de folhas.

Para a variável de massa seca da parte aérea (MMSPA), observou diferença apenas na adubação com cama de frango quando comparado com e sem a inoculação de sementes com B. japonicum. Já para as adubações, o tratamento com esterco bovino apresentou os melhores resultados, sendo seguido pelas adubações com cama de frango, adubo químico e testemunha em ambos os casos, com ou sem inoculação.

Conforme mencionado anteriormente, os compostos orgânicos utilizados possuem um grande aporte de $\mathrm{P}$ e K. $6,91 \%$ de $\mathrm{P}$ e $2,20 \%$ de $\mathrm{K}$ para o esterco bovino e $4,21 \%$, de $\mathrm{P}$ e $3,38 \%$ de $\mathrm{K}$ para a cama de frango. O que se pode concluir é que o maior acúmulo de massa seca de parte aérea em esterco bovino, seguido da cama de frango, é devido tanto a composição de $\mathrm{P}$ e K nesses compostos orgânicos quanto aos benefícios indiretos dessa adubação, uma vez que a utilização de adubos orgânicos aumenta os teores de matéria orgânica do solo, melhorando a sua fertilidade, favorecem a fauna microbiológica, que por sua vez atua na decomposição da matéria orgânica liberando nutrientes, além de melhorar as características do solo e o processo de nodulação (FALCÃO et al., 2013).

\section{CONCLUSÃO}

A adubação orgânica aliada com a inoculação de sementes, quando feitas corretamente, proporciona resultados satisfatórios ao meio de produção, relacionado ao desenvolvimento de plantas.

O esterco bovino é uma excelente escolha para a adubação de soja, possibilitando um maior número de nódulos, desenvolvimento radicular e de parte aérea, contribuindo para um maior rendimento no final do ciclo da cultura.

\section{REFERÊNCIAS}

AGUIAR, A. T. E.; GONÇALVES, C.; PATERNIANI, M. E. A. G. Z.; SANT'ANNA TUCCI, M. L.; CASTRO, C. E. F. (ed.). Boletim 200: Instruções agrícolas para as principais culturas econômicas. 7. ed. Campinas: Instituto Agronômico, 2014. 452 p.

ALCÂNTARA NETO, F.; PETTER, F. A.; PAVAN, B. E.; SCHMITT, C. R.; ALMEIDA, F. A.; PACHECO, L. P. PIAUILINO, A. C. Desempenho agronômico de cultivares de soja em duas épocas de semeadura no cerrado piauiense. Comunicata Scientiae, v. 3, n. 3, p.215-219, 2012.

AMARO, G. B. Adubos e fertilizantes. Brasília: Embrapa, 2007.

BÁRBARO, I. M.; MACHADO, P. C.; BÁRBARO JUNIOR, L. S.; TICELli, M.; MIGUEL, F. B.; SIlvA, J. A. A. Produtividade da soja em resposta á inoculação padrão e coinoculação. Colloquium Agrariae, p.01-07, 15, 2009. 10.5747/ca.2009.v05.n1.a0040.

BOSSOLANI, J. W.; POLONI, N. M.; LAZARINI, E.; BETTIOL, J. V. T.; FISCHER FILHO, J. A.; NEGRISOLI, M. M.et al. Development of RR soybean in function of glyphosate doses and Bradyrhizobium inoculation. Revista
Brasileira de Engenharia Agrícola e Ambiental, v. 22, n. 12, p.854-858, 2018. 10.1590/1807-1929/agriambi.v22n12p854$\underline{858}$.

BRACCINI, A. L.; MARIUCCI, G. E. G.; SUZUKAWA, A. K.; LIMA, L. H. S.; PICCININ, G.G. Co-inoculação e modos de aplicação de Bradyrhizobium japonicum e Bzospirillum brasilense e adubação nitrogenada na nodulação das plantas e rendimento da cultura da soja. Scientia Agraria Paranaensis, Marechal Cândido Rondon, v. 15, n. 1, p. 27-35, 2016.

CANATtO, R. A.; AgUiAR, L. F.; JUSTINO, G. C.; SOUZA, L. A.; CAMARGOS, L. S. O nitrato influencia o metabolismo de compostos nitrogenados em calopogônio (Calopogonium mucunoides) ao longo do ciclo de vida. Biotemas, v. 27, n. 1, p.1-8, 18, 2013. 10.5007/2175$\underline{7925.2014 \mathrm{v} 27 \mathrm{n} 1 \mathrm{p} 1 .}$

CARDOSO, A. I. I.; FERREIRA, K. P.; VIEIRA JÚNIOR, R. M.; ALCARDE, C. Alterações em propriedades do solo adubado com composto orgânico e efeito na qualidade das sementes de alface. Horticultura Brasileira, v. 29, n. 4, p.594599, 2011. 10.1590/s0102-05362011000400025.

CARVALHO, E. R.; RESENDE, P. M.; ANDRADE, M. J. B.; PASSOS, A. M. A.; OLIVEIRA, J. A. Fertilizante mineral e resíduo orgânico sobre características agronômicas da soja e nutrientes no solo. Revista Ciência Agronômica, v. 42, n. 4, p.930-939, 2011. 10.1590/s1806-66902011000400015.

CONAB. Companhia Nacional de Abastecimento. Acompanhamento da safra brasileira: grãos. Brasília: CONAB, v. 5, n. 3, dezembro de 2017. Disponível em: <https://www.conab.gov.br/info-agro/safras/graos/boletimda-safra-de-graos?start=10>. Acesso em: 5 out. 2018.

CONAB. Companhia Nacional de Abastecimento. Perspectivas para a agropecuária. Brasília: Conab, 2018. 104 p.

FAGAN, Evandro Binotto et al. Fisiologia da Fixação Biológica do Nitrogênio em Soja - Revisão. Revista da Fzva, Uruguaiana, v. 14, n. 1, p.89-106, 2007.

FALCÃO, J. V.; LACERDA, M. P. C.; MENDES, I. C.; LEÃO, T. P.; CARMO, F. F. Qualidade do solo cultivado com morangueiro sob manejo convencional e orgânico. Pesquisa Agropecuária Tropical, v. 43, n. 4, p.450-459, 2013. 10.1590/s1983-40632013000400004.

FERREIRA, D. F. Sisvar: a computer statistical analysis system. Ciência e Agrotecnologia (UFLA), v. 35, n.6, p. 1039-1042, 2011.

HUNGRIA, M.; NOGUEIRA, M. A.; ARAUJO, R.S. Coinoculation of soybeans and common beans with rhizobia and azospirilla: strategies to improve sustainability. Biology And Fertility Of Soils, v. 49, n. 7, p.791-801, 12, 2013. 10.1007/s00374-012-0771-5.

HUNGRIA, M.; CAMPO, R. J.; MENDES, I. C. A importância do processo de fixação biológica de nitrogênio para a cultura da soja: componente essencial para a 
competitividade do produto brasileiro. Londrina: Embrapa Soja, 2007. 80 p.

MELCHIORRE, M.; LUCA, M. J.; GONZALAZ ANTA, G.; SUAREZ, P.; LOPEZ, C.; LASCANO, R.; RACCA, R. W. Evaluation of bradyrhizobia strains isolated from field-grown soybean plants in Argentina as improved inoculants. Biology And Fertility Of Soils, v. 47, n. 1, p.81-89, 2010. 10.1007/s00374-010-0503-7.

NUNES, A. R. A.; FERNANDES, A. M.; LEONEL, M.; GARCIA, E. L.; MARGOLBO, L. A.; CARMO, E. L. Nitrogênio no crescimento da planta e na qualidade de raízes da mandioquinha-salsa. Ciência Rural, v. 46, n. 2, p.242-247, 2016. 10.1590/0103-8478cr20150339.

OLIVEIRA, A. P.; SILVA, V. R. F.; SANTOS, C. S.; ARAÚJO, J. S.; NASCIMENTO, J. T. Produção de coentro cultivado com esterco bovino e adubação mineral. Horticultura Brasileira, Brasília, v. 20, n. 3, p. 477-479, 2002.

PAMPANA, S.; MASONI, A.; MARIOTTI, M.; ERCOLI, L.; ARDUINI, I. Nitrogen fixation of grain legumes differs in response to nitrogen fertilisation. Experimental Agriculture, v. 54, n. 01, p.66-82, 2016. 10.1017/s0014479716000685.

PEDROZO, A.; OLIVEIRA, N. J. G.; ALBERTON, O. Biological nitrogen fixation and agronomic features of soybean (Glycine max (L.) Merr.) crop under different doses of inoculant. Acta Agronómica, v. 67, n. 2, p.297-302, 2018. $\underline{10.15446 / \text { acag.v67n2.56375. }}$

PEREIRA, M. G.; SANTOS, C. E. R. S., FREITAS, A. D. S.; STAMFORD, N. P.; ROCHA, G. S. D. C.; BARBOSA, A. T. Interactions between arbuscular mycorrhizal fungi Rhizobium and actinomycetes in the rhizosphere of soybean. Revista Brasileira de Engenharia Agrícola e Ambiental, v. 17, n. 12, p.1249-1256, 2013.

RAGAGNIN, V. A.; SENA JÚNIOR, D. G.; DIAS, D. S.; BRAGA, W. F.; NOGUEIRA, P. D. M. Growth and nodulation of soybean plants fertilized with poultry litter. Ciência e Agrotecnologia, v. 37, n. 1, p.17-24, 2013. 10.1590/s1413-70542013000100002.

RAIJ, B.; CANTARELLA, H.; QUAGGIO, J. A.; FURLANI, A. M. C. (ed.). Recomendações de adubação e calagem para o estado de São Paulo. 2. ed. rev. e atual. Piracicaba: Ceres; Potafos, 1997. 42p.

SANTOS, A. C. M.; CARNEIRO, J. S. S.; LEITE, R. C.; SOUZA, M. A. S.; FREITAS, G. A.; SILVA, R. R. Adubação fosfatada com fertilizante Basiduo ${ }^{\circledR}$ na cultura da soja no oeste da Bahia. Journal Of Bioenergy And Food Science, v. 2, n. 3, p.82-90, 2015. 10.18067/jbfs.v2i3.25.

SILVA, A. F.; CARVALHO, M. A. C.; SCHONINGER, E. L.; MONTEIRO, S.; CAIONE, G.; SANTOS, P. A. Doses de inoculante e nitrogênio na semeadura da soja em área de primeiro cultivo. Bioscience Journal, Umberlândia, v. 27, n. 3, p.404-412, 2011.
SOARES, M. M.; SEDIYAMA, T. S.; NEVES, J. C. L. SANTOS JUNIOR, H. C.; SILVA, L. J. Nodulation, Growth and Soybean Yield in Response to Seed Coating and Split Application of Phosphorus. Journal Of Seed Science, v. 38, n. 1, p.30-40, 2016. 10.1590/2317-1545v38n1155355.

SOUZA, L. H.; NOVAIS, R. F.; ALVAREZ, V. H.; VILLANI, E. M. A. Efeito do pH do solo rizosférico e não rizosférico de plantas de soja inoculadas com Bradyrhizobium japonicum na absorção de boro, cobre, ferro, manganês e zinco. Revista Brasileira de Ciência do Solo, v. 34, n. 5, p.1641-1652, 2010. 10.1590/s0100-06832010000500017.

VAN RAIJ, B.; CANTARELLA, H. Q.; ATONIO, J.; FURLANI, Â. M. C. (Ed.). Recomendações e Adubação e Calagem para o Estado de São Paulo. 2. ed. Campinas: Embrapa, 1997. 285p.

XAVIER, T. F.; ARAUJO, A. S. F.; SANTOS, V. B.; CAMPOS, F. L. Inoculação e adubação nitrogenada sobre a nodulação e a produtividade de grãos de feijão-caupi. Ciência Rural, v. 38, n. 7, p.2037-2041, 2008. 10.1590/s010384782008000700038.

ZILLI, J. É.; PEREIRA, G. M. D.; FRANÇA JÚNIOR, I.; SILVA, K.; HUNGRIA, M.; ROUWS, J. R. C. Dinâmica de rizóbios em solo do cerrado de Roraima durante o período de estiagem. Acta Amazonica, v. 43, n. 2, p.153-160, 2013. 10.1590/s0044-59672013000200004. 